S. Kobayashi

Nagoya Math. J.

Vol. 57 (1975), 153-166

\title{
NEGATIVE VECTOR BUNDLES AND COMPLEX FINSLER STRUCTURES
}

\author{
SHOSHICHI KOBAYASHI*)
}

\section{Introduction}

A complex Finsler structure $F$ on a complex manifold $M$ is a function on the tangent bundle $T(M)$ with the following properties. (We denote a point of $T(M)$ symbolically by $(z, \zeta)$, where $z$ represents the base coordinate and $\zeta$ the fibre coordinate.)

$$
\begin{aligned}
& F \text { is smooth outside of the zero section of } T(M) ;^{1)} \\
& F(z, \zeta) \geqq 0 \text { and }=0 \text { if and only if } \zeta=0 ; \\
& F(z, \lambda \zeta)=|\lambda|^{2} \cdot F(z, \zeta) \quad \text { for any complex number } \lambda .
\end{aligned}
$$

The geometry of complex Finsler structures was first studied by Rizza [8]. In [9], Rund explained the significance of (1.3) in detail and derived the connection coefficients and the equation of the geodesics. In this paper we study complex Finsler structures in holomorphic vector bundles. It is known that a holomorphic vector bundle is ample (in the sense of algebraic geometry) if it admits a hermitian metric of positive curvature. ${ }^{2}$ The converse is probably not true in general (except, of course, in the case of line bundles). We prove that a holomorphic vector bundle is negative ${ }^{3)}$ (i.e., its dual is ample) if and only if it admits a complex Finsler metric of negative curvature, thus making it possible to introduce differential geometric techniques into the study of ample and negative vector bundles.

Received September 25, 1974.

*) Supported partially by NSF Grant GP-42020X. This paper, presented to "Differentialgeometrie im Grossen" at Oberwolfach in June of 1974, was prepared during my stay in Oberwolfach and Bonn. I would like to express my gratitude to Professors Barner, Hirzebruch and Klingenberg who made this possible.

1) $F$ will be hermitian if it is smooth everywhere.

2) See Griffiths [3] and Kobayashi-Ochiai [6]. We use the term "ample" in the sense of Hartshorne [4].

3) Our negativity coincides with Grauert's weak negativity [2]. 


\section{Definitions and basic constructions}

Let $E$ be a holomorphic vector bundle of rank (i.e., fibre dimension) $r$ over a complex manifold $M$ of dimension $n$. The projective bundle $P(E)$ associated with $E$ is defined as follows. Let $E^{0}$ be the set of nonzero elements of $E$ and let $C^{*}=C-\{0\}$ act on $E^{0}$ by scalar multiplication. Then

$$
P(E)=E^{0} / C^{*}
$$

Geometrically, a point of $P(E)$ over $x \in M$ represents a complex line in the fibre $E_{x}$ of $E$ at $x$. Thus, $P(E)$ is a fibre bundle over $M$ with fibre $P_{r-1}(C)$, the complex projective space of dimension $r-1$. We organize various spaces and maps by the following diagram:

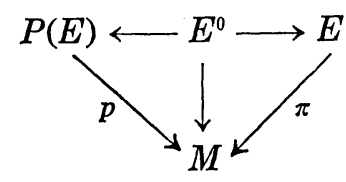

We denote the induced vector bundle $p^{-1} E$ over $P(E)$ by $\tilde{E}$ so that we have the following diagram:

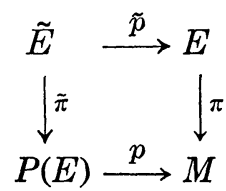

We consider the following tautological line subbundle $L$ of $\tilde{E}$. Given a point of $P(E)$, which represents a complex line in a certain fibre of $E$, the fibre of $L$ over that point is precisely the corresponding complex line. In other words, if $\tilde{x} \in P(E)$, then $L_{\tilde{x}}$ is a complex line in $\tilde{E}_{\tilde{x}}$ such that $\tilde{x}$ represents the line $\widetilde{p}\left(L_{\tilde{x}}\right)$. Let $L^{0}$ be the set of nonzero elements of $L$. Then, $\tilde{p}$ maps $L^{0}$ biholomorphically onto $E^{0}$. In other words, $L$ can be obtained from $E$ by blowing up the zero section of $E$ to $P(E)$.

In order to perform explicit local calculations, we use a local coordinate system $z=\left(z^{1}, \ldots, z^{n}\right)$ in $M$ and a system of linearly independent local holomorphic sections $s_{1}, \cdots, s_{r}$ of $E$. The dual frame of $s_{1}, \cdots, s_{r}$ will be denoted by $\zeta=\left(\zeta^{1}, \cdots, \zeta^{r}\right)$ so that $(z, \zeta)=\left(z^{1}, \cdots, z^{n}, \zeta^{1}, \cdots, \zeta^{r}\right)$ can be taken as a local coordinate system in $E$. When $\zeta \neq 0$, the point of $P(E)$ represented by $(z, \zeta)$ will be denoted by $(z,[\zeta])$. Putting 


$$
Z^{i}=\zeta^{i} \circ p
$$

we take $(z, \zeta, Z)=\left(z^{1}, \cdots, z^{n}, \zeta^{1}, \cdots, \zeta^{r}, Z^{1}, \cdots, Z^{r}\right)$ as a local coordinate system for $\tilde{E}$. Then the tautological line subbundle $L$ of $\tilde{E}$ is defined by the equations :

$$
Z^{i}=\zeta^{i} \quad \text { for } i=1, \cdots, r .
$$

A complex Finsler structure in $E$ is a real valued function $F$ on $E$ satisfying the following conditions:

(2.6) $F$ is smooth outside of the zero section of $E$, (i.e., $F$ is sufficiently differentiable in $E^{0}$ );

$$
\begin{aligned}
& F(z, \zeta) \geqq 0 \text { and }=0 \text { if and only if } \zeta=0 ; \\
& F(z, \lambda \zeta)=|\lambda|^{2} F(z, \zeta) \quad \text { for all } \lambda \in C .
\end{aligned}
$$

A holomorphic vector bundle $E$ with a complex Finsler structure $F$ is called a complex Finsler vector bundle.

Given a complex Finsler structure $F$, we set

$$
\begin{gathered}
F_{i j}=\partial^{2} F / \partial \zeta^{i} \partial \bar{\zeta}^{j} \quad \text { for } i, j=1, \cdots, r, \\
\tilde{F}(z, \zeta, Z)=\sum_{i, j} F_{i j}(z, \zeta) Z^{i} \bar{Z}^{j} .
\end{gathered}
$$

Then, as we shall see in the next section, we have

$$
F(z, \zeta)=\tilde{F}(z, \zeta, \zeta)
$$

that is, restricted to $L^{0}=E^{0}$, the function $\tilde{F}$ coincides with $F$. In general, there is no guarantee that $\left(F_{i j}\right)>0$ or even $\operatorname{det}\left(F_{i j}\right) \neq 0$. When $\left(F_{i j}\right)$ $>0$ (positive definite), $\tilde{F}$ defines a hermitian structure in the vector bundle $\tilde{E}$. In this case, we say that the Finsler structure $F$ is convex.

We note that if $F$ is a hermitian metric in $E$, it can be written locally in the following form:

$$
F(z, \zeta)=\sum g_{i j}(z) \zeta^{i \bar{\zeta}^{j}}
$$

so that

$$
F_{i j}=g_{i j}
$$

\section{Finsler connections}

We shall first derive some consequences of the homogeneity condition (2.8) following Rund [9]. We write 


$$
F_{i}=\partial F / \partial \zeta^{i}, \quad F_{j}=\partial F / \partial \bar{\zeta}^{j}, \quad F_{i j}=\partial^{2} F / \partial \zeta^{i} \partial \bar{\zeta}^{j} \text {, etc. }
$$

Differentiating (2.8) with respect to $\lambda$ and $\bar{\lambda}$ and then setting $\lambda=1$, we obtain

$$
\begin{gathered}
F(z, \zeta)=\sum F_{i}(z, \zeta) \zeta^{i}=\sum F_{j}(z, \zeta) \bar{\zeta}^{j} \\
F(z, \zeta)=\sum F_{i j}(z, \zeta) \zeta^{i \zeta^{j}} \\
F_{i j}(z, \lambda \zeta)=F_{i j}(z, \zeta)
\end{gathered}
$$

Differentiating (3.4) with respect to $\lambda$ and $\bar{\lambda}$ and then setting $\lambda=1$, we obtain

$$
\sum F_{i j k}(z, \zeta) \zeta^{k}=\sum F_{i j \bar{\varepsilon}}(z, \zeta) \bar{\zeta}^{\ell}=0 \text {. }
$$

Note that (3.3) proves (2.11). These identities will be used in calculating the connection coefficients and the curvature.

In the remainder of this section, we shall assume that the Finsler structure $F$ is convex, i.e., the matrix $\left(F_{i j}\right)$ is positive definite. Then $\tilde{F}=\tilde{F}(z, \zeta, Z)=\sum F_{i j}(z, \zeta) Z^{i} \bar{Z}^{j}$ defines a hermitian structure in the vector bundle $\tilde{E}$. We can therefore consider the hermitian connection of $\tilde{E}$ defined by $\tilde{F}$.

Let $\left(F^{i j}\right)$ be the inverse matrix of $\left(F_{i j}\right)$. Then the connection coefficients of the hermitian connection defined by $\tilde{F}$ are given by

$$
\begin{aligned}
& C_{j k}^{i}=\sum F^{i \hbar} \frac{\partial F_{j \hbar}}{\partial \zeta^{k}}, \\
& \Gamma_{j \beta}^{i}=\sum F^{i \hbar} \frac{\partial F_{j \hbar}}{\partial z^{\beta}} .
\end{aligned}
$$

From (3.5) we obtain

$$
\sum C_{j k}^{i} \zeta^{k}=0
$$

The local cross sections $s_{1}, \cdots, s_{r}$ of $E$ considered in $\S 2$ can be regarded in a natural manner as local cross sections of the induced bundle $\tilde{E}$. Then the covariant differential $\nabla s_{i}$ of these sections $s_{i}$ are defined by

$$
\nabla s_{i}=\sum \Gamma_{i \beta}^{k} s_{k} d z^{\beta}+\sum C_{i j}^{k} s_{k} d \zeta^{j}
$$

The hermitian connection of $\tilde{E}$ defined by (3.9) is called the Finsler connection of $E$ (defined by $F$ ). Its connection form $\left(\omega_{i}^{j}\right)$ is given by 


$$
\omega_{i}^{j}=\sum \Gamma_{i \beta}^{j} d z^{\beta}+\sum C_{i k}^{j} d \zeta^{k}
$$

so that

$$
\nabla s_{i}=\sum \omega_{i}^{j} s_{j}
$$

Its curvature form $\left(\Omega_{i}^{j}\right)$ is given by

$$
\Omega_{i}^{j}=d \omega_{i}^{j}+\sum \omega_{k}^{j} \wedge \omega_{i}^{k}=\bar{\partial} \omega_{i}^{j} .
$$

We can write

$$
\begin{aligned}
\Omega_{i}^{j}= & \sum R_{i \alpha \beta}^{j} d z^{\alpha} \wedge d \bar{z}^{\beta}+\sum P_{i \alpha \bar{\ell}}^{j} d z^{\alpha} \wedge d \bar{\zeta}^{\ell} \\
& +\sum P_{i k \beta}^{j} d \zeta^{k} \wedge d \bar{z}^{\beta}+\sum Q_{i k \bar{\ell}}^{j} d \zeta^{k} \wedge d \bar{\zeta}^{\ell},
\end{aligned}
$$

where

$$
\begin{array}{ll}
R_{i \alpha \bar{\beta}}^{j}=-\partial \Gamma_{i \alpha}^{j} / \partial \bar{z}^{\beta}, & P_{i \alpha \bar{\ell}}^{j}=-\partial \Gamma_{i \alpha}^{j} / \partial \bar{\zeta}^{\ell}, \\
P_{i k \beta}^{j}=-\partial C_{i k}^{j} / \partial \bar{z}^{\beta}, & Q_{i k \bar{\ell}}^{j}=-\partial C_{i k}^{j} / \partial \bar{\zeta}^{\ell} .
\end{array}
$$

Setting $R_{i j \alpha \beta}=\sum F_{k j} R_{i \alpha \beta}^{k}$, etc., we obtain from (3.6), (3.7) and (3.14) the following formulas:

$$
\begin{aligned}
& R_{i j \alpha \bar{\beta}}=-F_{i j \alpha \bar{\beta}}+\sum F^{k \bar{\ell}} F_{k \bar{\beta}} F_{i \bar{\ell} \alpha}, \\
& P_{i j \alpha \bar{l}}=-F_{i j \alpha \bar{\ell}}+\sum F^{k \hbar} F_{k j \bar{l}} F_{i \hbar \alpha}, \\
& P_{i j k \bar{\beta}}=-F_{i j k \bar{\beta}}+\sum F^{h \bar{\ell}} F_{h j \bar{\beta}} F_{i \bar{\ell} k}, \\
& Q_{i j k \bar{l}}=-F_{i j k \bar{l}}+\sum F^{h m} F_{h j \bar{l}} F_{i m k} .
\end{aligned}
$$

In the special case where $F$ is a hermitian metric, we have

$$
C_{i k}^{j}=P_{i j \alpha \overline{\bar{b}}}=P_{i j k \bar{\beta}}=Q_{i j k \bar{b}}=0 \text {. }
$$

Going back to the general case of a Finsler structure, to each unit vector $(z, \zeta, Z) \in \tilde{E},\left(\sum F_{i j}(z, \zeta) Z^{i} \bar{Z}^{j}=1\right)$, we associate the hermitian form defined by

$$
\begin{aligned}
& \sum R_{i j \alpha \beta} Z^{i} \bar{Z}^{j} d z^{\alpha} d \bar{z}^{\beta}+\sum P_{i j \alpha \bar{\xi}} Z^{i} \bar{Z}^{j} d z^{\alpha} d \bar{\zeta}^{\ell} \\
& \quad+\sum P_{i j k \beta} Z^{i} \bar{Z}^{j} d \zeta^{k} d \bar{z}^{\beta}+\sum Q_{i j k \ell} Z^{i} \bar{Z}^{j} d \zeta^{k} d \bar{\zeta}{ }^{\ell} .
\end{aligned}
$$

We are particularly interested in the case where this unit vector is contained in the line subbundle $L$, that is, the case where

$$
Z^{i}=\zeta^{i} / F(z, \zeta)^{1 / 2}
$$

Then, by (3.5) and (3.15) the expression in (3.17) reduces to 


$$
\Psi=\frac{1}{F(z, \zeta)} \sum R_{i j \alpha \beta} \zeta^{i \bar{\zeta}^{\jmath}} d z^{\alpha} d \bar{z}^{\beta}
$$

When $F$ is a hermitian metric, its curvature is said to be positive (resp. negative) if the form $\Psi$ in (3.19) is positive-definite (resp. negativedefinite), see Griffiths [3], Kobayashi-Ochiai [6]. If, moreover, $E$ is the tangent bundle $T(M)$ of a complex manifold $M$ with a hermitian metric, then $\Psi$ is positive-definite (resp. negative-definite) if and only if the socalled holomorphic bisectional curvature is positive (resp. negative), see Goldberg-Kobayashi [1]. It is therefore appropriate to say that the curvature of the convex complex Finsler structure $F$ in a vector bundle $E$ is positive (resp. negative) if $\Psi$ is positive-definite (resp. negativedefinite).

For the computational purpose, it is sometimes convenient to choose a suitable system of local holomorphic sections $s_{1}, \cdots, s_{r}$ of $E$. Given a point $a \in E^{0}$ (i.e., a nonzero element of $E$ ) or the corresponding point $[a] \in P(E)$ (i.e., the complex line through $a$ ), we can find suitable local holomorphic sections $s_{1}, \cdots, s_{r}$ of $E$ such that

$$
F_{i j}(a)=\delta_{i j}
$$

and

$$
F_{i j \alpha}(a)=0 \text {. }
$$

Since (3.21) is equivalent to the vanishing of $\Gamma_{i \alpha}^{j}$ at $a$, we refer to $(z, \zeta)$ $=\left(z^{1}, \cdots, z^{n}, \zeta^{1}, \cdots, \zeta^{r}\right)$ as a normal coordinate system around the point $a$. The existence of $s_{1}, \cdots, s_{r}$ with properties (3.20) and (3.21) is easily established by applying a suitable transformation $\left(f_{i}^{j}(z)\right)$ to an arbitrarily given system $t_{1}, \cdots, t_{r}$ of linearly independent local holomorphic sections. In fact, if we write

$$
s_{i}(z)=\sum f_{i}^{j}(z) t_{j}(z) \text { with } f_{i}^{j}(z)=c_{i}^{j}+\sum c_{i \alpha}^{j} z^{\alpha},
$$

then we can determine the constants $c_{i}^{j}, c_{i \alpha}^{j}$ by direct calculation.

\section{Finsler structures of negative curvature}

As in $\S 2$, let $E$ be a holomorphic vector bundle over $M, P(E)$ the associated projective bundle over $M, \tilde{E}$ the induced vector bundle over $P(E)$ and $L$ the tautological line subbundle of $\tilde{E}$. Because of the natural identification of $E^{0}$ ( $=E$ minus the zero section) and $L^{0}$ ( $=L$ minus the 
zero section), any function $F$ defined on $E^{0}$ may be considered as a function $h$ on $L^{0}$, and vice-versa. In particular, each Finsler structure $F$ in $E$ defines a hermitian structure $h$ in $L$, and vice-versa. (It is easy to verify that even though $F$ is not necessarily smooth along the zero section of $E, h$ is smooth everywhere on $L$ since it defines an inner product in each fibre of $L$ ). This establishes a natural one-to-one correspondence between the Finsler structures in $E$ and the hermitian structures in $L$.

We shall now calculate the connection form and the curvature form of $h$. Since $L^{0}$ may be considered as the principal bundle associated to $L$, we shall do our calculation on $L^{0}\left(=E^{0}\right)$. Then the connection form $\varphi$ (defined on $L^{0}$ ) is given by

$$
\varphi=\partial \log h
$$

and the curvature form is given by

$$
\Phi=\bar{\partial} \partial \log h .
$$

We set

$$
\begin{aligned}
\Phi= & \sum K_{\alpha \bar{\beta}} d z^{\alpha} \wedge d \bar{z}^{\beta}+\sum K_{\alpha j} d z^{\alpha} \wedge d \bar{\zeta}^{j} \\
& +\sum K_{i \bar{\beta}} d \zeta^{i} \wedge d \bar{z}^{\beta}+\sum K_{i j} d \zeta^{i} \wedge d \bar{\zeta}^{j}
\end{aligned}
$$

We shall now express these coefficients of the curvature form in terms of partial derivatives of $F$. Because of the identification $L^{0}=E^{0}$, we can replace $h$ in (4.1) and (4.2) by $F$. As in $\S 3$, we indicate partial derivatives of $F$ by subscripts. Thus, $F_{i j \alpha}=\partial^{3} F / \partial \zeta^{i} \partial \bar{\zeta}^{j} \partial z^{\alpha}$, etc. Then

$$
\begin{gathered}
K_{\alpha \bar{\beta}}=-\frac{1}{F} \sum F_{i j \alpha \bar{\beta}} \zeta^{i \bar{\zeta}^{j}}+\frac{1}{F^{2}} \sum F_{i j \alpha} F_{k \bar{\ell} \bar{\beta}} \zeta^{i \bar{\zeta}^{j} \zeta^{k \bar{\zeta}^{\ell}}} \\
K_{\alpha \bar{j}}=-\frac{1}{F} \sum F_{i j \alpha} \zeta^{i}+\frac{1}{F^{2}} \sum F_{j} F_{k \bar{\ell} \alpha} \zeta^{k \bar{\zeta}^{\ell}} \\
K_{i \bar{\beta}}=\bar{K}_{\beta \bar{i}}, \\
K_{i j}=-\frac{1}{F} F_{i j}+\frac{1}{F^{2}} \sum F_{i \bar{b}} F_{k j} \zeta^{k \bar{\zeta}^{\ell}}
\end{gathered}
$$

Considered as a form defined on $L^{0}$, the curvature form $\Phi$ is degenerate in the fibre direction since it is really a form on the base manifold $P(E)$ of the principal bundle $L^{0}$. Assume that $\Phi$ is negative as a form on $P(E)$, i.e., 


$$
\Phi(t, \bar{t})<0
$$

for every tangent vector $t$ of type $(1,0)$ of $L^{0}$ which is not vertical. In other words, assume that the matrix

$$
\left(\begin{array}{ll}
K_{\alpha \beta}(z, \zeta) & K_{\alpha j}(z, \zeta) \\
K_{i \beta}(z, \zeta) & K_{i j}(z, \zeta)
\end{array}\right)
$$

has $n+r-1$ negative eigen-values and one eigen-value equal to zero corresponding to the direction $\zeta$. In particular, the submatrix

$$
\left(K_{i j}(z, \zeta)\right)
$$

has $r-1$ negative eigen-values and one eigen-value equal to zero. From (4.7) it follows that $\left(F_{i j}\right)$ is positive-definite. Thus, we have proved:

LEMMA. If a hermitian structure $h$ in $L$ has negative curvature, then the corresponding Finsler structure $F$ in $E$ is convex.

Assume that the curvature of $h$ is negative. Since $\left(F_{i j}\right)$ is positivedefinite, we obtain a hermitian metric $\tilde{F}$ in $\tilde{E}$ as in $\S 2$. We shall now show that the curvature $\Psi$ of $F$ defined by (3.19) is negative. For this purpose, we make use of a normal coordinate system $(z, \zeta)$ as explained in $\S 3$. At the origin $\left(z_{0}, \zeta_{0}\right)$ of the coordinate system, (4.4)-(4.7) reduce to

$$
\begin{aligned}
& K_{\alpha \bar{\beta}}\left(z_{0}, \zeta_{0}\right)=-\frac{1}{F\left(z_{0}, \zeta_{0}\right)} \sum F_{i j \alpha \beta}\left(z_{0}, \zeta_{0}\right) \zeta_{0}^{i \bar{\zeta}_{0}^{j}}, \\
& K_{\alpha j}\left(z_{0}, \zeta_{0}\right)=K_{i \beta}\left(z_{0}, \zeta_{0}\right)=0, \\
& K_{i j}\left(z_{0}, \zeta_{0}\right)=-\frac{1}{F\left(z_{0}, \zeta_{0}\right)} \delta_{i j}+\frac{1}{F\left(z_{0}, \zeta_{0}\right)^{2}} \zeta^{j \bar{\zeta}^{i}} .
\end{aligned}
$$

Then the following conditions are equivalent:

(a) the curvature of $h$ is negative at $\left(z_{0},\left[\zeta_{0}\right]\right) \in P(E)$;

(b) the matrix (4.9) has $n+r-1$ negative eigenvalues (and one eigenvalue equal to zero) at $\left(z_{0}, \zeta_{0}\right)$;

(c) (i) the matrix (4.10) has $r-1$ negative eigen-values (and one eigen-value equal to zero) at $\left(z_{0}, \zeta_{0}\right)$, and (ii) the matrix $\left(K_{\alpha \beta}\left(z_{0}, \zeta_{0}\right)\right)$ is negative-definite.

We already know that (c.i) is equivalent to the convexity of $F$. To see the significance of (c.ii), we consider $R_{i j \alpha \beta}$ in terms of the normal coordinate system. From (3.15) we obtain 


$$
R_{i j \alpha \beta}\left(z_{0}, \zeta_{0}\right)=-F_{i j \alpha \beta}\left(z_{0}, \zeta_{0}\right) .
$$

From (3.19), (4.11) and (4.12), we obtain

$$
\Psi=\sum K_{\alpha \beta}\left(z_{0}, \zeta_{0}\right) d z^{\alpha} d \bar{z}^{\beta}
$$

at the origin $\left(z_{0}, \zeta_{0}\right)$. Thus, (c.ii) is equivalent to the condition that the curvature $\Psi$ of $F$ is negative. We have proved:

THEOREM 4.1. Let $E$ be a holomorphic vector bundle over $M$ and $L$ the tautological line bundle over $P(E)$. Let $F$ be a complex Finsler structure in $E$ and $h$ the corresponding hermitian structure in $L$. Then $F$ has negative curvature if and only if $h$ has negative curvature.

\section{Positive (i.e., ample) and negative bundles}

First, we make a few remarks on the bundle constructions of $\S 2$. The fibre $P(E)_{x}$ is the projective space of complex lines in $E_{x}$. Attaching the projective space $P^{*}\left(E_{x}\right)$ of hyperplanes in $E_{x}$ to each point $x$ of $M$, we obtain another projective bundle $P^{*}(E)$ over $M$. Since the hyperplanes in $E_{x}$ are in a natural one-to-one correspondence with the lines in the dual space $E_{x}^{*}$, the bundle $P^{*}(E)$ is naturally isomorphic to $P\left(E^{*}\right)$, where $E^{*}$ is the dual vector bundle of $E$.

We denote the induced vector bundle $\tilde{E}$ and the tautological line bundle $L$ over $P(E)$ of $\S 2$ by $\tilde{E}_{P(E)}$ and $L_{P(E)}$ to indicate their base space. Similarly, we denote the pull-back of $E$ and $E^{*}$ to $P\left(E^{*}\right)$ by $\tilde{E}_{P\left(E^{*}\right)}$ and $\tilde{E}_{P\left(E^{*}\right)}$ to indicate their base space $P\left(E^{*}\right)$. To each point $u$ of $P^{*}(E)\left(=P\left(E^{*}\right)\right)$ we attach the hyperplane of $\left(\tilde{E}_{P\left(E^{*}\right)}\right)_{u}$ corresponding to $u$. In this way we obtain a subbundle $S_{P\left(E^{*}\right)}$ of $\tilde{E}_{P\left(E^{*}\right)}$ of rank $r-1$. We denote the quotient bundle $\tilde{E}_{P\left(E^{*}\right)} / S_{P_{\left(E^{*}\right)}}$ by $H=H_{P\left(E^{*}\right)}$; it is a line bundle over $P\left(E^{*}\right)$. It is easy to verify that the tautological line bundle $L_{P\left(E^{*}\right)}$ over $P\left(E^{*}\right)$ constructed from $E^{*}$ is dual to $H=H_{P\left(E^{*}\right)}$.

In general, a line bundle $G$ over a compact complex manifold $V$ is said to be ample or positive if there is a positive integer $m$ such that $G^{m}$ is very ample in the sense that there are enough holomorphic sections of $G^{m}$ to induce an imbedding of $V$ into a projective space $P_{N}(C)$, (where $N+1$ is the dimension of the space $\Gamma\left(G^{m}\right)$ of holomorphic sections). It is a well-known theorem of Kodaira that $G$ is ample if and only if $G$ admits a hermitian structure of positive curvature. We say that $G$ is negative if its dual $G^{*}\left(=G^{-1}\right)$ is ample. A simple calculation 
shows that a hermitian structure in $G$ has negative curvature if and only if the corresponding dual hermitian structure in $G^{*}$ has positive curvature. Hence, $G$ is negative if and only if it admits a hermitian structure of negative curvature.

Let $E$ be a holomorphic vector bundle over $M$. As in [6], we say that $E$ is negative if the tautological line bundle $L=L_{P(E)}$ is negative. On the other hand, $E$ is said to be ample or positive if the line bundle $H=H_{P\left(E^{*}\right)}$ is ample. From the fact that $H_{P\left(E^{*}\right)}$ is dual to $L_{P\left(E^{*}\right)}$, it follows that $E$ is ample if and only if its dual bundle $E^{*}$ is negative. From Theorem 4.1 we obtain immediately.

THEOREM 5.1. A holomorphic vector bundle $E$ over a compact complex manifold $M$ is negative if and only if it admits a convex complex Finsler structure of negative curvature.

Problem. It is reasonable to expect that $E$ is ample if and only if it admits a complex Finsler structure of positive curvature. The question is whether $E$ admits a complex Finsler structure of positive curvature if and only if $E^{*}$ admits a complex Finsler structure of negative curvature. Given a complex Finsler structure $F$ in $E$, let $F^{*}$ be the complex Finsler structure in $E^{*}$ defined by

$$
F^{*}\left(z, \zeta^{*}\right)=\sup _{F(z, \zeta)=1}\left|\left\langle\zeta^{*}, \zeta\right\rangle\right|^{2},
$$

where the supremum is taken over all $\zeta \in E_{z}$ such that $F(z, \zeta)=1$. It is very likely that $F$ has positive curvature if and only if $F^{*}$ has negative curvature. The difficulty lies in finding more computable relationships between $F$ and $F^{*}$.

It may be appropriate to raise here another question with difficulties of similar nature. It is known that if $E_{1}$ and $E_{2}$ are two ample vector bundles over $M$, then $E_{1} \otimes E_{2}$ is ample, see Hartshorne [4]. The proof is highly non-trivial. One wonders if this global statement can be proved locally by a differential geometric method. Since it is easier to treat negative bundles than ample bundles from our view point, we consider two negative vector bundles $E_{1}$ and $E_{2}$ over $M$ with complex Finsler structures $F_{1}$ and $F_{2}$, respectively, of negative curvature. Let $F$ be the naturally induced complex Finsler structure in $E=E_{1} \otimes E_{2}$. The question is whether $F$ has also negative curvature. According to the theory 
of normed vector spaces, the naturally induced Finsler structure $F$ should be defined by

$$
F(z, \zeta)=\inf \sum_{i, j} F_{1}\left(z, \zeta_{i}^{\prime}\right) \cdot F\left(z, \zeta_{j}^{\prime \prime}\right)
$$

where the infimum is taken over all possible ways of expressing $\zeta$ as $\zeta=\sum \zeta_{i}^{\prime} \otimes \zeta_{j}^{\prime \prime}$ in $E_{z}$, see Schaeffer $[11 ;$ p. 93]. Then

$$
F\left(z, \zeta^{\prime} \otimes \zeta^{\prime \prime}\right)=F_{1}\left(z, \zeta^{\prime}\right) \cdot F_{2}\left(z, \zeta^{\prime \prime}\right) .
$$

Again, the problem seems to be the difficulty of expressing $F$ in terms of $F_{1}$ and $F_{2}$ in a more computable way.

\section{Subbundles and curvature}

\section{Let}

$$
0 \rightarrow E^{\prime} \rightarrow E \rightarrow E^{\prime \prime} \rightarrow 0
$$

be an exact sequence of holomorphic vector bundles. We know (see Hartshorne [4]) that if $E$ is ample, so is $E^{\prime \prime}$. By dualizing this fact, we know that if $E$ is negative, so is $E^{\prime}$. We shall show differential geometrically that $E^{\prime}$ is more negative than $E$.

We choose local holomorphic sections $s_{1}, \cdots, s_{r}$ of $E$ in such a way that $s_{1}, \cdots, s_{p}$ are sections of $E^{\prime}$, where $p$ is the rank (=fibre dimension) of $E^{\prime}$. Then $E^{\prime}$ is locally defined by

$$
\zeta^{p+1}=\cdots=\zeta^{r}=0 .
$$

Let $F$ be a complex Finsler structure in $E$ and $F^{\prime}$ its restriction to $E^{\prime}$. From (3.3), we obtain

$$
F^{\prime}(z, \zeta)=\sum_{i, j=1}^{p} F_{i j}(z, \zeta) \zeta^{i \bar{\zeta}^{j}}
$$

If $\left(F_{i j}(z, \zeta)\right)_{1 \leqq i, j \leqq r}$ is positive definite, so is the principal minor $\left(F_{i j}(z, \zeta)\right)_{1 \leqq i, j \leqq p}$. Hence, if $F$ is convex, so is $F^{\prime}$. We shall now assume that $F$ is convex. Using the first equation of (3.15), we compare the curvature $R_{i j \alpha \bar{\beta}}^{\prime}$ of $F^{\prime}$ with the curvature $R_{i j \alpha \bar{\beta}}$ of $F$. (In view of the definition (3.19), we are not interested in the remaining components of the curvature). As in $\S 3$, given a nonzero element $a$ of $E^{\prime}$ we can choose a suitable system $s_{1}, \cdots, s_{r}$ of holomorphic sections such that

$$
\begin{array}{ll}
F_{i j}(a)=\delta_{i j} & \text { for } i, j=1, \cdots, r, \\
F_{i j \alpha}(a)=0 & \text { for } 1 \leqq i \leqq p<j \leqq r \text { and all } \alpha .
\end{array}
$$


The proof is similar to the one we gave in $\S 3$ to obtain $s_{1}, \ldots, s_{r}$ satisfying (3.20) and (3.21). But we are now under the additional restriction that $s_{1}, \cdots, s_{p}$ be sections of $E^{\prime}$. This imposes the condition

$$
f_{i}^{j}=0 \quad \text { for } 1 \leqq j \leqq p<i \leqq r
$$

on the transformation $\left(f_{i}^{j}(z)\right)$ used in (3.22). It is because of the restriction (6.5) that we get only (6.4) instead of (3.20) and (3.21). Now we have

$$
R_{i j \alpha \bar{\beta}}^{\prime}=R_{i j \alpha \bar{\beta}}-\sum_{k=1}^{p} F_{k j \alpha} F_{i \hbar \bar{\beta}} \quad \text { for } i, j=1, \cdots, p
$$

at the point $a$ of $E^{\prime}$. Hence,

THEOREM 6.1. Let $E$ be a holomorphic vector bundle with a convex complex Finsler structure $F$. Let $E^{\prime}$ be a subbundle of $E$ and $F^{\prime}$ the restriction of $F$ to $E^{\prime}$. Then $F^{\prime}$ is also convex, and its curvature does not exceed the curvature of $F$.

Consider the case where we have a convex complex Finsler structure $F$ in the tangent bundle $T(M)$ of a complex manifold $M$. Let $N$ be a complex submanifold of $M$. Set $E=\left.T(M)\right|_{N}$ and $E^{\prime}=T(N)$. Applying Theorem 6.1 to this situation, we see that the curvature of $N$ does not exceed the curvature of $M$.

In the case of the tangent bundle $T(M)$, we can define, in addition to the curvature defined in (3.19), the holomorphic sectional curvature:

$$
\frac{1}{F(z, \zeta)^{2}} \sum R_{i j k \bar{\zeta}}{ }^{i} \bar{\zeta}^{\top} \zeta^{k} \bar{\zeta}^{\ell}
$$

which is a function on the projective bundle $P(T(M))$. We see also immediately that the holomorphic sectional curvature of a complex submanifold does not exceed that of $M$. As in the hermitian case (see Kobayashi [5]) we have the following

THEOREM 6.2. If $M$ is a complex manifold with a convex complex Finsler structure $F$ whose holomorphic sectional curvature is bounded from above by a negative constant, then $M$ is hyperbolic.

From Theorems 5.1 and 6.2, we obtain

COROLLARY 6.3. Let $M$ be a compact complex manifold with negative tangent bundle (i.e., with ample cotangent bundle). Then $M$ is hyperbolic. 


\section{Vanishing of sections}

It is well known that a negative line bundle over a compact manifold has no non-trivial sections. We prove

THEOREM 7.1. Let $E$ be a holomorphic vector bundle over a compact complex manifold $M$. If $E$ admits a convex complex Finsler structure $F$ whose curvature form $\Psi$ (defined by (3.19)) has at least one negative eigen-value at every point $(z,[\zeta])$ of $P(E)$ (and so, in particular, if $E$ is a negative bundle), then $E$ has no holomorphic sections other than the zero section.

The assumption in the theorem means that the hermitian matrix

$$
\left(\sum R_{i j \alpha \beta}(z,[\zeta]) \zeta^{i \bar{\zeta}^{j}}\right)_{\alpha, \beta=1, \cdots, n}
$$

has at least one negative eigen-value.

Proof. This is a straightforward generalization of the hermitian case (see Kobayashi-Wu [7]). Assuming that $E$ has a non-trivial holomorphic section $\zeta(z)$, consider the function

$$
f(z)=F(z, \zeta(z))
$$

on $M$. Let $z_{0}$ be one of the points where $f$ attains its maximum and set $\zeta_{0}=\zeta\left(z_{0}\right)$. Composed with the projection $P(E) \rightarrow M, f$ may be considered as a function on $P(E)$ taking its maximum at $\left(z_{0},\left[\zeta_{0}\right]\right) \in P(E)$. We denote by $\nabla_{\alpha}$ and $\nabla_{\beta}$ the covariant differentiation by $z^{\alpha}$ and $\bar{z}^{\beta}$ with respect to the hermitian connection of bundle $\tilde{E}$ defined by the hermitian metric $\tilde{F}$. Considering $\zeta(z)$ as a section $\tilde{E}$ in a natural manner, we calculate the complex Hessian

$$
f_{\alpha \beta}=\nabla_{\beta} \nabla_{\alpha} f, \quad \alpha, \beta=1, \cdots, n .
$$

Since $\zeta(z)=\sum \zeta^{i}(z) s_{i}(z)$ is holomorphic, we have

$$
\nabla_{\beta} \zeta^{i}=0 \text {. }
$$

Hence, the Ricci identity reduces to

$$
\nabla_{\bar{\beta}} \nabla_{\alpha} \zeta^{i}=-\sum R_{j \alpha \bar{\beta}}^{i} \zeta^{j} .
$$

From (7.5) we obtain 


$$
\begin{aligned}
f_{\alpha \bar{\beta}} & =\nabla_{\beta} \nabla_{\alpha}\left(\sum F_{i j} \zeta^{i \bar{\zeta}^{j}}\right) \\
& =\sum F_{i j} \nabla_{\beta} \nabla_{\alpha} \zeta^{i} \cdot \bar{\zeta}^{j}+\sum F_{i j} \nabla_{\alpha} \zeta^{i} \cdot \nabla_{\beta} \bar{\zeta}^{j} \\
& =-\sum R_{i j \alpha \beta} \zeta^{i \bar{\zeta}^{j}}+\sum F_{i j} \nabla_{\alpha} \zeta^{i} \cdot \nabla_{\beta} \bar{\zeta}^{j} .
\end{aligned}
$$

This shows that $\left(f_{\alpha \beta}\right)$ has always at least one positive eigen-value. On the other hand, at $z_{0}$ where $f$ attains its maximum, that is impossible. This completes the proof.

A similar argument proves that if $E$ admits a convex complex Finsler structure $F$ whose curvature form $\Psi$ has at least one positive eigen-value at every point of $P(E)$, then every holomorphic section $\zeta(z)$ of $E$ has zeros. (Assuming that $\zeta(z)$ has no zeros, calculate the complex Hessian of $f$ at one of the minimum points).

\section{BIBLIOGRAPHY}

[1] S. I. Goldberg and S. Kobayashi, On holomorphic bisectional curvature, J. Differential Geometry 1 (1967), 225-233.

[2] H. Grauert, Über Modifikationen und exzeptionelle analytische Mengen, Math. Annalen 146 (1962), 331-368.

[ 3 ] P. A. Griffiths, Hermitian differential geometry, Chern classes and positive vector bundles, Global Analysis in honor of Kodaira, 1969, 185-252.

[ 4 ] R. Hartshorne, Ample vector bundles, Publ. I. H. E. S. 29 (1966), 319-350.

[5] S. Kobayashi, Hyperbolic Manifolds and Holomorphic Mappings, Marcel Dekker, Inc. New York, 1970.

[6] S. Kobayashi and T. Ochiai, On complex manifolds with positive tangent bundles, J. Math. Soc. Japan 22 (1970), 499-525.

[ 7] S. Kobayashi and $\mathrm{H}$. Wu, On holomorphic sections of certain hermitian vector bundles, Math. Annalen 189 (1970), 1-4.

[ 8 ] G. B. Rizza, $F$-forme quadratiche ed hermitiane, Rend. Mat. e Appl. 23 (1965), 221-249.

[ 9 ] H. Rund, Generalized metrics on complex manifolds, Math. Nachrichten 34 (1967), $55-77$.

[10] H. Rund, The Differential Geometry of Finsler Spaces, Springer-Verlag, 1959.

[11] H. H. Schaeffer, Linear Topological Spaces, Macmillan, 1966.

University of California, Berkeley 